\title{
Preventive Effect of Electrical Stimulation Biofeedback Combined With Family Individualized Pelvic Floor Rehabilitation Training on Postpartum Pelvic Floor Dysfunction
}

\author{
Huan Wang ${ }^{1}$, , Hong Zhou ${ }^{2}$, Li Cheng ${ }^{2}$ \\ ${ }^{1}$ Office of Out-patient, The First Affiliated Hospital of Jinan University, Guangzhou, China \\ ${ }^{2}$ Outpatient Department of Obstetrics and Gynecology, The First Affiliated Hospital of Jinan University, Guangzhou, China
}

Email address:

54214880@qq.com (Huan Wang)

${ }^{*}$ Corresponding author

\section{To cite this article:}

Huan Wang, Hong Zhou, Li Cheng. Preventive Effect of Electrical Stimulation Biofeedback Combined With Family Individualized Pelvic Floor Rehabilitation Training on Postpartum Pelvic Floor Dysfunction. Journal of Surgery. Vol. 8, No. 2, 2020, pp. 43-47. doi: $10.11648 /$ j.js.20200802.11

Received: February 1, 2020; Accepted: February 17, 2020; Published: March 6, 2020

\begin{abstract}
Objective: To explore the preventive effect of electrical stimulation biofeedback combined with family individualized pelvic floor rehabilitation training on postpartum pelvic floor dysfunction (PFD). Methods: From June 2018 to June 2019, 124 women who underwent the first postpartum review (Postpartum4-6 weeks, lochia clean, no vaginal bleeding) in our hospital were randomly divided into observation group and control group, 62 cases in each group. The control group received routine pelvic floor muscle training intervention, while the observation group received family individualized pelvic floor rehabilitation training combined with electrical stimulation biofeedback intervention. Six months after the intervention, the pelvic floor muscular fibre strength and A3 reflex, pelvic organ prolapse quantitative (POP-Q) score, pelvic floor dysfunction questionnaire (PFDI20), pelvic floor disease quality of life questionnaire (PFIQ7) and pelvic organ prolapse, urinary incontinence function questionnaire (PISQ-12) were compared between the two groups. Results: After 6 months of intervention, there was no significant difference in the muscle strength of type I muscle fibers between the two groups $(\mathrm{Z}=-0.918, \mathrm{P}=0.358)$, while the muscle strength of type II muscle fibers in the observation group was significantly better than that in the control group $(\mathrm{Z}=-2.372, \mathrm{P}=0.018)$. There was no significant difference in $\mathrm{A} 3$ reflex between the two groups before and after treatment (before: $\chi^{2}=0.387, \mathrm{P}=0.534$; after: $\left.\chi^{2}=0.683, \mathrm{P}=0.409\right)$. The POP-Q score of the observation group was significantly better than that of the control group $(\mathrm{Z}=-2.073, \mathrm{P}=0.038)$. There was no significant difference in PFDI20, PFIQ7 and PISQ-12 scores between the two groups before and after treatment $(\mathrm{P}>005)$. In the observation group, there were 2 cases of vaginal relaxation, 1 case of mild uterine prolapse, no stress urinary incontinence and vaginal wall bulge, the incidence was $4.84 \%$. In the control group, 4 cases had vaginal relaxation, 2 cases had mild uterine prolapse, 1 case had stress urinary incontinence and no vaginal wall bulge, the incidence was $11.29 \%$. Conclusion: Electrical stimulation biofeedback combined with family individualized pelvic floor rehabilitation training has a better effect on pelvic floor muscle rehabilitation, which is helpful to prevent the occurrence of PFD.
\end{abstract}

Keywords: Electrical Stimulation Biofeedback, Individualization, Pelvic Floor Rehabilitation Training, Postpartum, Pelvic Floor Dysfunction Disease

\section{Introduction}

Pelvic floor dysfunction (pelvic floor dysfunction, PFD) is a group of gynecological diseases caused by pelvic supporting structure damage or dysfunction, including stress urinary incontinence, pelvic floor organ prolapse and sexual dysfunction [1]. Previous studies have shown that the decrease of pelvic floor muscle strength is the influencing factor of PFD, and delivery leads to the injury of pelvic floor supporting tissue in varying degrees [2]. Therefore, early postpartum 
screening of pelvic floor function and active intervention can reduce the incidence of PFD and improve the quality of life [3] At present, the commonly used clinical rehabilitation methods of pelvic floor function are pelvic floor muscle exercise and electrical stimulation biofeedback, both of which have achieved good results [4-6]. The rehabilitation of pelvic floor function is a long-term process, and the continuous management of patients after discharge and return to their families is of great significance to the prevention of PFD. However, affected by psychological, social, environmental and other factors, patients still have problems such as improper training methods and poor compliance. Therefore, it is very important to develop a personalized overall rehabilitation plan for continuous management according to the situation of patients. The biofeedback method of electrical stimulation has the advantages of safety, reliability, painlessness and economy. Patients are easy to accept and can take the initiative to continue self-training [6]. A randomized controlled study was conducted on 124 parturients to observe the changes of physiological indexes and quality of life in order to explore the preventive effect of electrical stimulation biofeedback combined with family individualized pelvic floor rehabilitation training on postpartum pelvic floor dysfunction.

\section{Materials and Methods}

\subsection{Object of Study}

From June 2018 to June 2019, 124 women who underwent the first postpartum reexamination in our hospital (4-6 weeks postpartum, clean lochia, no vaginal bleeding) were studied, aged 19-42 years. Inclusion criteria: (1) Full-term natural delivery, singleton parturient; (2) Normal cognitive function, can cooperate with the evaluation of relevant indicators; (3) There are no pregnancy complications or delivery complications. Exclusion criteria: (1) Previous history of pelvic surgery; (2) History of pelvic organ prolapse and urinary incontinence before pregnancy; (3) Complicated with long-term chronic cough or constipation. 124 women were randomly divided into observation group $(n=62)$ and control group $(n=62)$. This study has been approved by the Ethics Committee of our hospital, and all the subjects signed the informed consent form.

\subsection{Methods}

\subsubsection{Methods of Rehabilitation}

The control group was treated with routine pelvic floor muscle training. Group education was used to explain the clinical manifestations and causes of postpartum pelvic floor disorders and pelvic floor training methods. Patients were taught to do Kegel exercises at home: take horizontal position, separate legs, tighten urethra, tighten anus, contract pelvic floor muscles for 5 seconds, relax for 5 seconds, 15 minutes for each training, and do 3 rapid contractions at the end of practice. Do exercises twice a day, gradually increase the frequency and intensity according to the training situation. All parturients were followed up by telephone after discharge, once every 2 weeks in the first 3 months, once every 4 weeks in the last 3 months, and the effect of pelvic floor training was evaluated 6 months later. During the follow-up period, the daily training time and times were recorded.

The observation group was treated with family individualized pelvic floor rehabilitation training combined with electrical stimulation biofeedback intervention. First of all, the French PHENIX USB4 neuromuscular stimulation therapeutic apparatus (Guangzhou Shanshan Medical device Industry Co. Ltd.) was used for electrical stimulation biofeedback therapy. In the first step, the proprioceptive sensation of muscle contraction was awakened, the treatment frequency was adjusted to $50 \mathrm{~Hz}$, the pulse width was adjusted to $250 \mathrm{us}$, and the current was adjusted according to the maternal sensation; in the second step, the contractile function of class I muscle fibers was trained, the treatment frequency was adjusted to $832 \mathrm{~Hz}$, and the pulse width was adjusted to $300 \mathrm{~Hz} 750 \mathrm{~Hz}$; in the third step, the treatment frequency was adjusted to $20 \mathrm{~Hz}$, and the pulse width was adjusted to $20 \mathrm{~Hz}$. The fourth step is to train class I and II muscle fibers to provide training templates for parturients to train independently. Biofeedback therapy with electrical stimulation was performed 3 times a week, 20 minutes each time, for a total of 10 times. Four weeks later, the family individualized pelvic floor overall rehabilitation training was carried out on the basis of the control group; (1) The individualized pelvic floor muscle function training program was set up according to the specific conditions of the parturient: double diagnosis was used to guide the parturient to master the correct training methods. for example, guide patients to insert their fingers into the vagina and contract the pelvic floor muscles, if the contraction mode is correct, you can feel pressure around the fingers. (2) Instruct the parturient to avoid contracting the gluteus maximus and abdominal muscles in the process of training, so as not to induce pelvic floor muscle dysfunction. (3) Urge parturients to record the number and time of daily training in order to improve their training compliance. (4) To guide and supervise the gradual increment and gradual use of the pelvic floor rehabilitation device; (5) To reasonably customize individual rehabilitation exercises, such as rectus abdominis separation, vaginal relaxation and sexual dysfunction; and the solution of family individualized rehabilitation in abnormal situation. (6) Carry on the simple psychological counseling to alleviate the bad mood that the parturient may have. The parturients were followed up by telephone after electrical stimulation biofeedback intervention, once every 2 weeks in the first 3 months, once every 4 weeks in the last 3 months, and the effect of pelvic floor training was evaluated 6 months later. During the follow-up period, the daily training time and times of parturients were recorded, the basic psychological and physiological conditions of parturients were understood, and the parturients who failed to master training skills were given careful guidance.

\subsubsection{Obvervational Index}

The main results were as follows: (1) After baseline and 
intervention for 6 months, the muscle strength and A3 reflex of pelvic floor type I and II muscle fibers were measured with neuromuscular stimulation therapy apparatus. The muscle strength of contraction $0 \mathrm{~s}$ was 0 , lasting $1 \mathrm{~s}$ was grade $\mathrm{I}$, and by analogy, lasting $\geq 5 \mathrm{~s}$ was grade $\mathrm{V}$ (normal muscle strength) [7]. A3 reflex is a very important reflex in urine control, and abnormal A3 reflex exists in some patients with PFD [8]. (2) Quantitative (PelvicOrgan Prolaps Quantitation, POP-Q grading method was used to analyze the degree of pelvic organ prolapse. 0 degree: $\mathrm{Aa}, \mathrm{Ap}, \mathrm{Ba}, \mathrm{Bp}$ were all at- $3 \mathrm{~cm}, \mathrm{C}$ point or D point was at-tv1 - $(\operatorname{tv} 1 \sim 2) \mathrm{cm}$; stage I: the farthest end of prolapse was located at $<-1 \mathrm{~cm}$; degree II: the farthest end of prolapse was located at $-1 \mathrm{~cm} \sim 1 \mathrm{~cm}$; degree III: the farthest end of prolapse was located at $+1 \mathrm{~cm} \sim(\operatorname{tv} 1 \sim 2) \mathrm{cm}$;. Grade IV: the farthest end of prolapse is located at $>$ (full length of vagina-2) cm [9].(3) Quality of life evaluation: pelvic floor dysfunction questionnaire (Pelvic Floor Distress Inventory-shortform20, PFDI20), pelvic floor disease quality of life questionnaire short form (Pelvic Floor Impact Questionnaire shortform7,
PFIQ7) and pelvic organ prolapse and urinary incontinence sexual function questionnaire (Pelvic organ prolapse urinary incontinence sexual questionnaire-short form 12) [10].

\subsection{Statistical Method}

SPSS 20.0 was used for data analysis. T-test was used for the comparison of measurement data, 2-test was used for the comparison of counting data, and rank sum test was used for the comparison of grade data. The difference was statistically significant $(\mathrm{P}<0.05)$.

\section{Results}

\subsection{The General Information}

There was no significant difference in age, mode of delivery, neonatal body mass and maternal BMI between the two groups.

Table 1. Comparison of two groups of general data.

\begin{tabular}{|c|c|c|c|c|}
\hline Basic information & Observation group $(n=62)$ & control group $(n=62)$ & $\mathbf{t} / \chi^{2}$ & $\mathbf{P}$ \\
\hline Age $($ year, $\bar{x} \pm s)$ & $27.26 \pm 5.12$ & $26.33 \pm 6.03$ & 0.926 & 0.357 \\
\hline $\begin{array}{l}\text { BMI }(\mathrm{kg} / \mathrm{m} 2, \bar{x} \pm s) \\
\text { Delivery mode [Number of cases }(\%)\end{array}$ & $26.33 \pm 5.01$ & $27.36 \pm 6.94$ & 0.948 & 0.346 \\
\hline $\begin{array}{l}\text { Vaginal delivery } \\
\text { Cesarean section }\end{array}$ & $\begin{array}{l}43(69.35) \\
19(30.65)\end{array}$ & $\begin{array}{l}41(66.13) \\
21(33.87)\end{array}$ & 0.148 & 0.701 \\
\hline The weight of a newborn $(\mathrm{g}, \bar{x} \pm s)$ & $3296.35 \pm 265.01$ & $3286.33 \pm 345.25$ & 0.182 & 0.856 \\
\hline
\end{tabular}

\subsection{Comparison of Electrophysiological Indexes of Pelvic Floor Before and After Intervention}

There was no significant difference in the muscle strength of class I and class II muscle fibers between the two groups before intervention $(\mathrm{Z}=-0.076, \mathrm{P}=0.939 ; \mathrm{Z}=-0.439, \mathrm{P}=0.661$ ). After 6 months of intervention, the muscle strength of class I muscle fibers and class II muscle fibers in the observation group were significantly improved $(Z=-2.668, P=0.008$; $\mathrm{Z}=-2.014, \mathrm{P}=0.044)$. After intervention in the control group, the muscle strength of type I muscle fiber was significantly improved $(Z=-1.965, P=0.049)$, but the muscle strength of type II muscle fiber was not significantly improved $(Z=-0.367$, $\mathrm{P}=0.713$ ). After 6 months of intervention, there was no significant difference in the muscle strength of class I muscle fibers between the two groups $(\mathrm{Z}=-0.918, \mathrm{P}=0.358)$, but the muscle strength of class II muscle fibers in the observation group was significantly better than that in the control group $(\mathrm{Z}=-2.372, \mathrm{P}=0.018)$. There was no significant difference in A3 reflex between the two groups before and after treatment (Before treatment $\chi^{2}=0.387, \quad \mathrm{P}=0.534$; After treatment $\chi^{2}=0.683, \mathrm{P}=0.409$ ), as shown in Table 2 .

Table 2. Comparison of muscle strength and A3 reflex of muscle fibers between the two groups [Number of cases (\%)].

\begin{tabular}{|c|c|c|c|c|}
\hline \multirow{2}{*}{ Project } & \multicolumn{2}{|c|}{ Observation group $(n=62)$} & \multicolumn{2}{|c|}{ Experimental group $(n=62)$} \\
\hline & Base line & After 6 months & Base line & After 6 months \\
\hline \multicolumn{5}{|c|}{ Class I muscle fiber } \\
\hline Grade 0 & $8(12.90)$ & $2(3.23)$ & $7(11.29)$ & $3(4.84)$ \\
\hline Grade I & $12(19.35)$ & $5(8.06)$ & $9(14.52)$ & $7(11.29)$ \\
\hline Grade II & $8(12.90)$ & $6(9.68)$ & $11(17.74)$ & $6(9.68)$ \\
\hline Grade III & $8(12.90)$ & $9(14.52)$ & $11(17.74)$ & $12(19.35)$ \\
\hline Grade IV & $9(14.52)$ & $16(25.81)$ & $9(14.52)$ & $13(20.97)$ \\
\hline \multicolumn{5}{|c|}{ Class II muscle fiber } \\
\hline Grade 0 & $6(9.68)$ & $2(3.23)$ & $7(11.29)$ & $6(9.68)$ \\
\hline Grade I & $6(9.68)$ & $6(9.68)$ & $6(9.68)$ & $5(8.06)$ \\
\hline Grade II & $11(17.74)$ & $5(8.06)$ & $10(16.13)$ & $8(12.90)$ \\
\hline Grade III & $8(12.90)$ & $3(4.84)$ & $9(14.52)$ & $12(19.35)$ \\
\hline Grade IV & $11(17.74)$ & $20(32.26)$ & $14(22.58)$ & $15(24.19)$ \\
\hline Grade V & $20(32.26)$ & $26(41.94)$ & $16(25.81)$ & $16(25.81)$ \\
\hline Abnormal & $14(22.58)$ & $6(9.68)$ & $17(27.42)$ & $9(14.52)$ \\
\hline
\end{tabular}




\subsection{Comparison of POP-Q Grading Between the Two Groups After Intervention}

After 6 months of intervention, the POP-Q score of the observation group was significantly better than that of the control group $(\mathrm{Z}=-2.073, \mathrm{P}=0.038)$.

Table 3. Comparison of POP-Q grading between the two groups after intervention [Number of cases (\%)].

\begin{tabular}{lll}
\hline Project & Observation group $(\mathbf{n}=\mathbf{6 2})$ & Experimental group $(\mathbf{n}=\mathbf{6 2})$ \\
\hline 0 degree & $48(77.42)$ & $39(62.90)$ \\
I degree & $10(16.13)$ & $9(14.52)$ \\
II degree & $3(4.84)$ & $9(14.52)$ \\
III degree & $1(1.61)$ & $4(6.45)$ \\
IV degree & 0 & $1(1.61)$ \\
\hline
\end{tabular}

\subsection{Comparison of Quality of Life and Quality of Sexual Life Between the Two Groups After Intervention}

There was no significant difference in PFDI20, PFIQ7 and PISQ-12 scores between the two groups before and after treatment (Comparison between groups before intervention:
PFDI20 $\mathrm{t}=1.183, \mathrm{P}=0.239$; PFIQ7 $\mathrm{t}=0.183, \mathrm{P}=0.856$; PISQ-12 $\mathrm{t}=0.177, \mathrm{P}=0.860$. Comparison between groups after 6 months: PFDI20 $\mathrm{t}=0.309, \mathrm{P}=0.758$; PFIQ7 $\mathrm{t}=1.409, \mathrm{P}=0.162$; PISQ-12 $\mathrm{t}=0.520, \mathrm{P}=0.604$ ), as shown in Table 4 .

Table 4. comparison of PFDI20, PFIQ7 and PISQ-12 scores between the two groups after intervention (score, $\bar{x} \pm s$ ).

\begin{tabular}{lllll}
\hline \multirow{2}{*}{ Project } & Observation group $(\mathbf{n}=\mathbf{6 2})$ & \multicolumn{2}{l}{ Experimental group $(\mathbf{n}=\mathbf{6 2})$} \\
\cline { 2 - 5 } & Baseline & After 6 months & Baseline & After 6 months \\
\hline PFDI20 & $7.36 \pm 1.02$ & $7.02 \pm 2.02$ & $7.40 \pm 0.85$ & $7.12 \pm 1.56$ \\
PFIQ7 & $30.31 \pm 10.33$ & $28.02 \pm 6.32$ & $30.58 \pm 5.38$ & $29.36 \pm 4.02$ \\
PISQ-12 & $8.03 \pm 0.89$ & $7.89 \pm 2.01$ & $8.06 \pm 1.00$ & $8.03 \pm 0.67$ \\
\hline
\end{tabular}

After 6 months of intervention, there were 2 cases of vaginal relaxation and 1 case of mild uterine prolapse in the observation group, no stress urinary incontinence and vaginal wall prolapse were found, and the incidence rate was $4.84 \%$. In the control group, vaginal relaxation occurred in 4 cases, mild uterine prolapse in 2 cases, stress urinary incontinence in 1 case, and no vaginal wall prolapse was found in 1 case (11.29\%).

\section{Discussion}

Pregnancy increases the volume and weight of the uterus, and the uterus is gradually in a vertical position, so that the pelvic floor tissue continues to be pressed and gradually relaxed. Changes in the levels of progesterone and estrogen during the perinatal period lead to dissolution of collagen in the pelvic floor ligament and tension relaxation of smooth muscle [11]. During delivery, the pelvic floor ligaments, fascia and muscles are overstretched or even broken. Transvaginal delivery is the influencing factor of pelvic floor structural injury, especially when the second stage of labor is prolonged, lateral episiotomy and instrumental delivery are more likely to occur [12]. Pregnancy and delivery may cause PFD by damaging the structure of the pelvis or changing the function of the pelvic floor muscles. The clinical manifestations of PFD are various, mainly stress urinary incontinence, pelvic floor organ prolapse and sexual dysfunction, which seriously affect the health and quality of life of parturients [13]. Therefore, it is very important to prevent the occurrence of PFD in the early stage. In this study, electrical stimulation biofeedback combined with family individualized pelvic floor rehabilitation training was used to carry out preventive intervention on PFD.

Electrical stimulation biofeedback is to place an electrode in the vagina and give an adjustable low-frequency electrical stimulation to improve the excitability of the pelvic floor muscle, exercise the muscle contraction, and then promote the functional recovery of the pelvic floor muscle. The biofeedback of electrical stimulation can divide the pelvic floor muscle fibers into type I and type II, and different frequency and pulse width stimulation can be given according to the different muscle fiber function of the patients, so it is more targeted than the traditional pelvic floor muscle training. Previous studies have shown that electrical stimulation biofeedback can increase the pelvic floor tension of parturients, and the effective rates are $83.5 \%$ and $81.4 \%$ at 6 and 12 months respectively [14]. Zhang Chengqiong et al. [15] found that electrical stimulation biofeedback can not only improve the pelvic floor muscle strength of PFD patients, but also down-regulate the expression levels of serum relaxin-2 and endothelin-1. Pelvic floor muscle exercise, also known as Kegel method, is one of the common methods of pelvic floor functional rehabilitation. Kegel method is to improve urethral and anal sphincter by consciously contracting pelvic floor muscles. It is clinically used in mild PFD, postpartum pelvic floor rehabilitation and various adjuvant therapy before and after pelvic floor surgery. This method has the advantages of economy and strong maneuverability, but its curative effect is uneven because of poor patient compliance and non-standard operation. Electrical stimulation biofeedback and Kegel method can complement each other and promote pelvic floor rehabilitation [18]. This study found that after intervention, 
the muscle strength of class II muscle fibers and the degree of POP-Q in the observation group were significantly better than those in the control group, indicating that the effect of pelvic floor muscle rehabilitation by electrical stimulation biofeedback combined with family individualized pelvic floor rehabilitation training was better. The rehabilitation of pelvic floor function is a long-term process, and the continuous management of patients after discharge and return to their families is of great significance to the prevention of PFD [19]. In this study, the family management of parturients was carried out from the aspects of specifying individual training program, telephone supervision and psychological counseling. After 6 months, $4.84 \%$ of PFD, occurred in the observation group and $11.29 \%$ in the control group, indicating that individualized pelvic floor rehabilitation training plays a positive role in the prevention of PFD.

\section{Conclusion}

To sum up, the results of this study show that electrical stimulation biofeedback combined with family individualized pelvic floor overall rehabilitation training has a better effect on pelvic floor muscle rehabilitation, which is helpful to prevent the occurrence of PFD. However, this study also has some limitations, such as relatively short follow-up time and long-term effect to be investigated. In addition, the sample size is relatively small, so it is necessary to carry out multicenter research and expand the sample size in the future to confirm the conclusions of this study.

\section{References}

[1] Dan Yang. Research progress of female pelvic floor dysfunction [J]. CHINESE JOURNAL OF FAMILY PLANNING \& GYNECOTOKOLOGY Volume 9 Number 2 2017: 11-15.

[2] Van Geelen H, Ostergard D, Sand P. A review of the impact of pregnancy and childbirth on pelvic floor function as assessed by objective measurement techniques [J]. Int Urogynecol J, 2018, 29 (3): 327-338.

[3] Schofield C, Newton RU, Cohen PA, et al. Health-related quality of life and pelvic floor dysfunction in advanced-stage ovarian cancer survivors: associations with objective activity behaviors and physiological characteristics [J]. Support Care Cancer, 2018, 26 (7): 2239-2246.

[4] Zhijing Sun, Lan Zhu, Jinghe Lang, et al. Postpartum pelvic floor rehabilitation on prevention of female pelvic floor dysfunction: a multicenter prospective randomized controlled study [J]. Chin J Obstet Gynecol, June 2015, Vol. 50, No. 6: 420-427.

[5] Rivalta M, Sighinolfi MC, Micali S, et al. Sexual function and quality of life in women with urinary incontinence treated by a complete pelvic floor rehabilitation program (biofeedback, functional electrical stimulation, pelvic floor muscles exercises, and vaginal cones) [J]. J Sex Med, 2010, 7 (3): 1200-1208.

[6] Yun Zhao. Effect of short-term postpartum biofeedback electrical stimulation on pelvic floor muscle strength [J]. Maternal and child health care in China, 2019, 34 (6): 123-126.

[7] Elmelund M, Biering-Sorensen F, Due U, et al. The effect of pelvic floor muscle training and intravaginal electrical stimulation on urinary incontinence in women with incomplete spinal cord injury: an investigator-blinded parallel randomized clinical trial [J]. Int Urogynecol J, 2018, 29 (11): 1597-1606.

[8] Yuanyuan Su, Yanhua Han, Li Cao. Pelvic electrophysiological evaluation in patients with pelvic organ prolapse before and after operation. [J]. Chinese Journal of practical Gynecology and Obstetrics, 2017, 33 (10): 1038-1040.

[9] ACOG Committee on Practice Bulletins--Gynecology. ACOG Practice Bulletin No. 85: Pelvic organ prolapse [J]. Obstet Gynecol, 2007, 110 (3): 717-729.

[10] Bilgic D, Gokyildiz S, Kizilkaya NB, et al. Quality of life and sexual function in obese women with pelvic floor dysfunction [J]. Women Health, 2018, 59 (1): 101-113.

[11] Hill AM, McPhail SM, Wilson JM, et al. Pregnant women's awareness, knowledge and beliefs about pelvic floor muscles: a cross-sectional survey [J]. Int Urogynecol J, 2017, 28 (10): 1557-1565.

[12] Zhao Y, Zou L, Xiao M, et al. Effect of different delivery modes on the short-term strength of the pelvic floor muscle in Chinese primipara [J]. BMC Pregnancy Childbirth, 2018, 18 (1): 275-283.

[13] Verbeek M, Hayward L. Pelvic floor dysfunction and its effect on quality of sexual life [J]. Sex Med Rev, 2019, 24 (1): 1-9.

[14] Guzman Rojas R, Wong V, Shek KL, et al. Impact of levator trauma on pelvic floor muscle function [J]. Int Urogynecol J, 2014, 25 (3): 375-380.

[15] Qiongcheng Zhang, Shan Yu, Jichun Shao. Influence of electrical stimulation biofeedback therapy on the pelvic floor muscle and serum related indexes of patients with pelvic floor dysfunction [J]. Journal of Clinical Medicine in Practice, 2016, 20 (15): 57-59.

[16] Lamin E, Parrillo LM, Newman DK, et al. Pelvic floor muscle training: underutilization in the USA [J]. Curr Urol Rep, 2016, 17 (2): 10-19.

[17] Nguyen MT, Armstrong AA, Wieslander CK, et al. Now anyone can kegel: one-time office teaching of pelvic floor muscle exercises [J]. Female Pelvic Med Reconstr Surg, 2019, 25 (2): 149-153.

[18] Yuqi Li, Haiyi Liu, Yuanyuan Wu, et al. Effect of biofeedback combined with electrical stimulation and Kegel training in the treatment of postpartum pelvic floor dysfunction [J]. Journal of Xinxiang Medical University, 2017, 34 (7): 619-622.

[19] Lin Cao, Shiqi Xiao, Lina Ge, et al. The influence of continuing care on long-term postoperative prognosis of pelvic floor dysfunction [J]. Modern Medical Journal. 2015, Jul; 43 (7): 891-896. 